\title{
Influence of Brettanomyces custersianus Upon the Activity of Saccharomyces cerevisiae Strains During the Tumultuous Phase of Vinification
}

\author{
Gildo Almeida da Silva ${ }^{1 *}$, Carolina Madalozzo Poletto ${ }^{2}$, Jandora Severo Poli ${ }^{2}$ and Patricia \\ Valente $^{3}$ \\ ${ }^{I}$ Departamento de Microbiologia; Embrapa Uva e Vinho; C. P.: 130; 95700-000; Bento Gonçalves - RS - Brasil. \\ ${ }^{2}$ Embrapa Uva e Vinho; 95700-000; Bento Gonçalves - RS - Brasil. ${ }^{3}$ Departamento de Microbiologia; Universidade \\ Federal do Rio Grande do Sul; Porto Alegre - RS - Brasil
}

\begin{abstract}
The aim of this work was to evaluate the influence of Brettanomyces custersianus on the metabolic activity of Saccharomyces cerevisiae during the tumultuous stage of wine production. The Cabernet Sauvignon grape must with the skin was inoculated with individual cultures of Sacch. cerevisiae and with mixed cultures of Sacch. cerevisiae and Br. custersianus. During the 6-day tumultuous phase of fermentation, the highest ethanol production and the highest sugar consumption were obtained with the strains without B. custersianus. Fermentations carried out with the addition of Brettanomyces metabolites, acetic acid and 4-ethylphenol, showed that only the former inhibited the growth of both Sacch. cerevisiae strains used. In some cases, Br. custersianus could affect the rate higher alcohols production and their final concentrations during the tumultuous phase of vinification.
\end{abstract}

Key words: Dekkera/Brettanomyces, wine, vinification, Custer effect

\section{INTRODUCTION}

The presence of Brettanomyces can devalue the quality of wine due to the production of offflavours such as horse sweat, Band Aid, barnyard, and burnt plastic. Not all the characteristics produced by Brettanomyces are universally disliked. This genus grows and survives in wine with high ethanol levels and low sugar supply. Silva et al. (2004) showed that ethanol can be used by $B r$. bruxellensis and Dekkera anomala as the sole carbon and energy source and that their growth was dependent on the concentration of alcohol in the culture media. Conterno et al. (2006) showed that only $26 \%$ of 35 Brettanomyces strains could grow on ethanol as a sole carbon source. The strains of Dekkera/Br. bruxellensis isolated by Conterno et al. (2006) could be distinguished in $\underline{\mathrm{a}}$ (European), $\underline{\mathrm{b}}$ (Americas) and $\underline{\mathrm{c}}$ (United States and New Zealand) groups according to $26 \mathrm{~S}$ rDNA and physiological aspects. The group b (Americas) is particularly difficult to control in winery environment due to both its high tolerance to $\mathrm{SO}_{2}$ and high level of 4-ethylphenol and 4-ethylguaiacol production. According to Renouf and Lonvaud-Funel (2007), the grape is

*Author for correspondence: gildo@ cnpuv.embrapa.br 
definitely the primary source of Brettanomyces and in being so, the prevention starts in the vineyard.

Microorganisms other than Dekkera or Brettanomyces also are volatile phenols formers. According to Chatonnet et al. (1997), among the lactic acid bacteria evaluated, Lactobacillus plantarum was the only bacterium capable of forming important quantities of 4-ethylphenol, a volatile phenol with a disagreeable odour of "wet horse". The amount produced by these bacteria was lower than that formed by Br. bruxellensis. The synthesis of volatile phenols by these bacteria is inhibited by procyanidic tannins. The synthesis of these compounds by $\mathrm{Br}$. bruxellensis is not affected by this polyphenolic compound found in red wines. The production of 4-ethylphenol and 4ethylguaiacol has been shown to be highly variable. Some strains produce large amount of both the compounds while other strains produce low amounts (Conterno et al., 2006). Therefore, the chromatographic analysis of 4-ethylphenol and 4-ethylguaiacol, used as a method to detect the presence of Brettanomyces in wine, may not be useful for many strains. Moreover, other compounds, such as tetrahydropyridines (Heresztyn, 1986) and acetic acid which negatively affect the analytical profile of the wine are produced by Brettanomyces. These compounds can not be detected by measuring 4-ethylphenol and 4-ethylguaiacol.

It should be stressed that $\mathrm{Br}$. custersianus is a negative Custer species (Scheffers and Wiken, 1969) and is also found in wine with the same frequency as Br. bruxellensis (Querol et al., 1990). This species does not produce acetic acid from glucose. This organic acid is formed when ethanol is used as the carbon or energy source (Freer et al., 2003). It is well known that high levels of acetic acid can be detrimental not only to wine quality as it imparts a vinegary/acetone-like aroma (Eglinton and Henschke, 1999a) but have also been associated with sluggish/stuck fermentations (Bisson, 1999) and with incomplete fermentation (Eglinton and Henschke, 1999). If this is true, $B r$. custersianus should not affect the Sacch. cerevisiae metabolism only at the beginning of the tumultuous phase of the vinification process, period in which the concentration of sugar is high and ethanol is low. The inhibition process should progressively increase as soon as the ethanol is formed.
The main purpose of this study was to evaluate the negative impact of $\mathrm{Br}$. custersianus on the metabolic activity of neutral and killer Sacch. cerevisiae strains during the tumultuous stage of the wine production and establish the point in which this impact definitely started.

\section{MATERIALS AND METHODS}

\section{Microorganism}

The neutral strain Saccharomyces cerevisiae Embrapa 1vvt/97, the killer strain Saccharomyces cerevisiae Embrapa 91B/84 and Brettanomyces custersianus NRRL Y-6653 were used. For single treatments the strains used were 1vvt/97 (T1) and 91B/84 (T2). For mixed cultures, the strains employed were 1vvt/97+Brett (Sacch. cerevisiae Embrapa 1vvt/97 and Br. custersianus NRRL Y6653) (T3) and 91B/84+Brett (Sacch. cerevisiae Embrapa 91B/84 and Br. custersianus NRRL Y6653) (T4). The experimental measurements were analysed as a completely randomised design with four treatments and three repetitions. The yeasts were maintained in G7 medium (da Silva and de Almeida, 2006) and must agar (da Silva, 1996).

\section{Inoculum}

The inocula were prepared in a must containing media as described by da Silva (1996). Six Fernbach flasks containing $1 \mathrm{~L}$ of culture medium were sterilised at $121^{\circ} \mathrm{C}$ for $30 \mathrm{~min}$. The medium was inoculated with the neutral strain Sacch. cerevisiae Embrapa 1vvt/97, the killer strain Sacch. cerevisiae Embrapa 91B/84 and $B r$. custersianus. The liquid cultures were incubated at $25^{\circ} \mathrm{C}$ and $150 \mathrm{rpm}$ in a rotatory shaker (New Brunswick G-27, Edison, N. J., USA). After 16 h of growth, $2 \mathrm{~L}$ of each strain containing media were aseptically transferred to three $20 \mathrm{~L}$ glass Carboys containing $10 \mathrm{~L}$ of must media. The system was aerated with $2 \mathrm{vvm}$ of filter sterilised air and maintained at $25^{\circ} \mathrm{C}$ for $24 \mathrm{~h}$. The air was sterilised by filtering through a $0.2 \mu \mathrm{m}$ Millipore (Millex-FG 50) filter. After 24h, the aeration system was stopped. Then a glass stopper in the bung-hole that allowed the gas to escape through a water trap was inserted at the top of the Carboys and this anaerobic process was maintained for 16 h. The cultures were centrifuged at $10,000 \mathrm{x}$ g for $15 \mathrm{~min}$ and the wet weight of the cells was determined. The initial amount of the cells added 
was equivalent to the amount present in $1.7 \mathrm{~L}$ of a culture $(10 \%$ v/v) of Sacch. cerevisiae Embrapa $1 \mathrm{vvt} / 97$ and Sacch. cerevisiae Embrapa 91B/84 for $17 \mathrm{~L}$ of grape must. The initial concentration of cells of the mixed cultures corresponded to the amount of cells present in $850 \mathrm{ml}$ of culture Sacch. cerevisiae and $850 \mathrm{ml}$ of $\mathrm{Br}$. custersianus.

\section{Tumultuous phase}

The grapes were passed through a mechanical destemmer-crusher to remove the stems and release the juice. The juice and the skins were transferred to 12 carboys (three carboys for each treatment). After inoculation, the cultures were maintained at $24^{\circ} \mathrm{C}$ during all stages of the vinification process. The tumultuous phase was performed with the skins and lasted 6 days.

Metabolic response of Sacch. cerevisiae to single and combined inoculation with $\mathrm{Br}$. custersianus in sterile grape must:

Test tubes containing $10 \mathrm{ml}$ of steam-sterilised grape must were inoculated with $1 \mathrm{ml}$ of a single cell suspension containing $10^{7} \mathrm{cell} / \mathrm{ml}$ of Sacch. cerevisiae 1vvt/97, Sacch. cerevisiae 91B/84 and Br. custersianus NRRL Y-6653. A mixed culture was performed with $0.5 \mathrm{ml}$ of a suspension containing $10^{7}$ cell $/ \mathrm{ml}$ of Sacch. cerevisiae 1vvt $/ 97$ and $0.5 \mathrm{ml}$ of Br. custersianus NRRL Y-6653. The another mixed culture was prepared with $0.5 \mathrm{ml}$ Sacch. cerevisiae $91 \mathrm{~B} / 84$ and $0.5 \mathrm{ml}$ of $\mathrm{Br}$. custersianus NRRL Y-6653. The metabolic activity of the microorganisms was measured by $\mathrm{CO}_{2}$ evolution according to Giudici and Zambonelli (1992). The experimental measurements were analysed as a completely randomised design with five treatments and five repetitions.

\section{Chemical Analysis}

Analysis of anaerobic metabolites

Aliquots of $100 \mathrm{ml}$ of medium were distilled and the original volume of the samples was filled up with distilled water to a volume of $100 \mathrm{ml}$. The ethanol was then determined by measuring the density of the distillate with an Anton-Paar DMA58 densitometer. The temperature of the sample was controlled with a water bath (Haag-G, West Germany). The concentration of acetaldehyde, ethylacetate and higher alcohols were measured by capillary gas chromatography (Perkin Elmer AutoSystem XL) equipped with a flame-ionisation detector (FID), a capillary column $(0.25 \mathrm{~mm}$ i.d. by $50 \mathrm{~m}$ - CPWAX57B), and a split/splitless injector. Splitless injections were made onto the column at $40^{\circ} \mathrm{C}(5$-min hold) with oven programming from $40^{\circ} \mathrm{C}\left(2^{\circ} \mathrm{C} / \mathrm{min}\right)$ to $60^{\circ} \mathrm{C}(10 \mathrm{~min})$, then $\left(15^{\circ} \mathrm{C} / \mathrm{min}\right)$ to $200^{\circ} \mathrm{C}(18 \mathrm{~min})$. The 4-methyl-pentanol-2 was used as internal standard and the separation was performed under a constant flow of $1.8 \mathrm{ml} \mathrm{He} / \mathrm{min}$ at $30 \mathrm{psi}$ head pressure, $85.7 \mathrm{ml}$ synthetic air $/ \mathrm{min}$ and $53.1 \mathrm{ml}$ $\mathrm{H}_{2} /$ min.

\section{Analysis of reducing sugar}

The reducing sugar and sucrose assays were done by the reduction of Fehling alkaline cupric solutions as described by Ribéreau-Gayon et al. (1982). When sucrose was used, hydrolytic procedure with $1 \mathrm{ml} \mathrm{HCl}$ was performed before the analysis.

\section{Volatile Acidity}

The volatile acidity was done by distillation as described by Ribéreau-Gayon et al. (1982). In short, the samples were steam distilled with Cazenave-Ferre equipment followed by titration with $\mathrm{NaOH}(0.1 \mathrm{~N})$. The results were expressed as $\mathrm{mEq} / \mathrm{L}$.

Inhibition of Sacch. cerevisiae by 4-ethyl-phenol and acetic acid

The assay for the detection of inhibition by 4ethylphenol was performed by preparing a stock solution containing 1g/L 4-ethylphenol (Merck). The stock solution was sterilised by filtering through a $0.45 \mu \mathrm{m}$ Millipore filter and added to grape must to give concentrations of 0 (control), 4.46, 9.90, 14.78 and $19.61 \mu \mathrm{g} / \mathrm{ml}$. The assay for the detection of inhibition by acetic acid was performed adding commercial acetic acid (Merck) to grape must to give concentrations of 0 (control), 9.90, 19.61, 29.13, 38.46 and $47.62 \mu \mathrm{L} / \mathrm{ml}$. The metabolic activity of both the assays was measured by $\mathrm{CO}_{2}$ evolution (Giudici and Zambonelli, 1992).

\section{Statistical analysis}

The regression analysis was performed by using $\mathrm{R}$ Program for Debian GNU/Linux (Venables et al., 2004; Logan, 2005). R version 2.7.1 (2008-06-23) Copyright (C) 2008 The $R$ Foundation for Statistical Computing. 


\section{RESULTS AND DISCUSSION}

Activity of Sacch. cerevisiae during tumultuous phase of vinification

The activity of Sacch. cerevisiae seemed to be repressed by the presence of Br. custersianus (Fig. 1). The statistical analysis with regard to $24 \mathrm{~h}$ consumption revealed no significant difference between the two single cultures (T1 and T2) and between the single culture Sacch. cerevisiae 91B/84 (T2) and the mixed culture Sacch. cerevisiae 1vvt/97+Brett (T3) $(\mathrm{P}>0.05)$. The difference between the two mixed cultures (T3 and T4) was significant $(\mathrm{P}<0.05)$. The other differences ( $\mathrm{T} 1$ and $\mathrm{T} 3, \mathrm{~T} 1$ and $\mathrm{T} 4, \mathrm{~T} 2$ and $\mathrm{T} 4$ ) were highly significant $(\mathrm{P}<0.01)$. This suggested that the reduced consumption of sugar in the mixed culture was not only due to the lower concentration of Sacch. cerevisiae at the time of inoculation in relation to the single cultures. The ethanol formation in $24 \mathrm{~h}$ was not significantly different between the two mixed cultures $(\mathrm{P}>0.05)$.

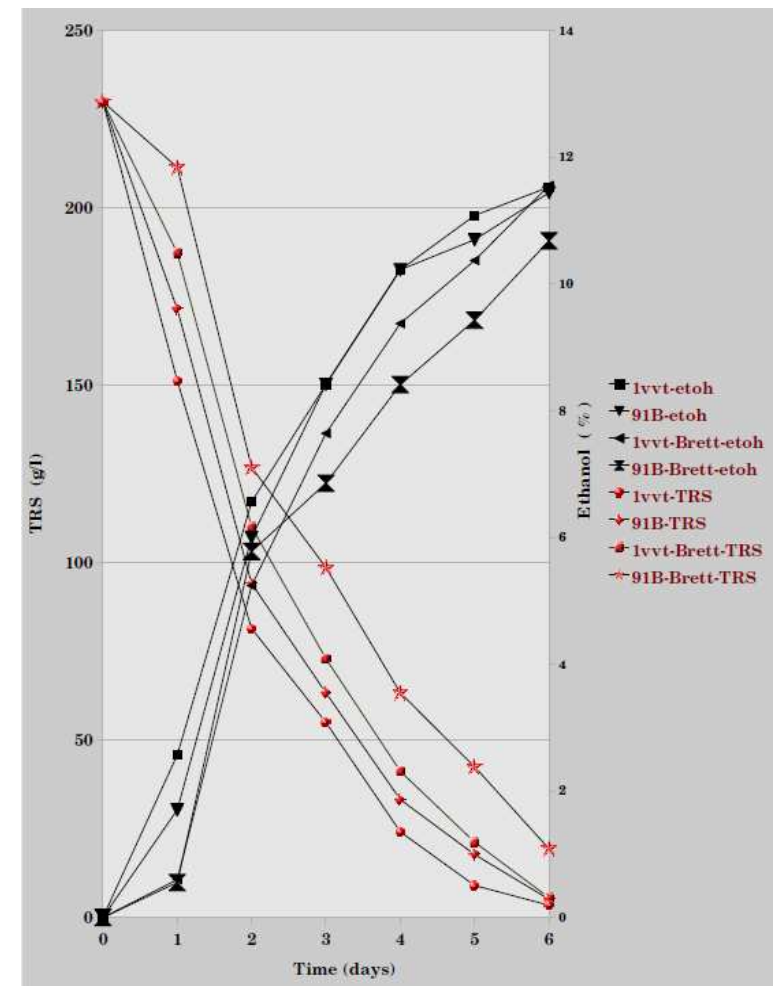

Figure 1 - Total reducing sugar (TRS) and ethanol (etoh) production by single culture of Sacch. cerevisiae 1vvt/97 and 91B/84 and mixed culture of Sacch. cerevisiae 1vvt/97 with Br. custersianus and Sacch. cerevisiae 91B/84 with Br. Custersianus.

The other differences were highly significant $(\mathrm{P}<0.01)$. The strain Sacch. cerevisiae 1vvt/97 proved to be metabolically more active than the strain Sacch. cerevisiae 91B/84 in producing ethanol only when $B r$. custersianus was not added. Taking the consumption and the ethanol formation into account, it was observed that in $24 \mathrm{~h}$ of fermentation, the metabolism of Sacch. cerevisiae $1 \mathrm{vvt} / 97$ in presence of $\mathrm{Br}$. custersianus was deviated to the products other than ethanol. In fact, the only significantly lower ethanol yield $\left(\mathrm{Y}_{\mathrm{p} / \mathrm{s}}\right)$ was obtained with the mixed culture Sacch. cerevisiae 1vvt/97 Br. custersianus $(\mathrm{P}<0.05)$. At the end of tumultuous phase, there was no significant differences between the treatments with regard to yield $(\mathrm{P}>0.05)$ but the ethanol produced at that time by the mixed culture Sacch. cerevisiae 91B/84+Brett was highly significant lower than those of treatment with either combination $(\mathrm{P}<0.01)$. This showed that Br. custersianus could cause impact on the metabolism of Sacch. cerevisiae $91 \mathrm{~B} / 84$. It should be considered that microorganisms other than Sacch. cerevisiae and $B r$. custersianus were present during the process of wine production. 
Higher alcohols are not less important compounds in the wine. With regard to these compounds, the two strains of Sacch. cerevisiae used presented different production rates. It was interesting to note that the strain 91B/84 had a higher linear rate of 3-methyl-1-butanol production than the strain $1 \mathrm{vvt} / 97$ (Table 1). The linear production rate was even higher in the presence of $\mathrm{Br}$. custersianus. The strain 1vvt/97 t presented lower production rate and also increased its synthesis in the presence of Br. custersianus. It seemed that this microorganism was a 3-methyl-1-butanol producer or an inductor of this higher alcohol. The linear rate of production 2-methyl-1-butanol was also higher for the strain 91B/84 than for $1 v v t / 97$. In the presence of $\mathrm{Br}$. custersianus, the strain 1vvt/97 increased its production rate, whereas the strain 91B/84 had its rate decreased (Table 1). As the rates with and without $B r$. custersianus were similar, the presence of this microorganism did not affect the linear rate of the production of 2-methyl1-propanol (Table 1), albeit the rate of production was higher with the strain 1vvt/97.

Table 1 - Linear regressions for 3-methyl-1-butanol and 2-methyl-1-butanol production between the third and the sixth day of tumultuous fermentation

\begin{tabular}{llll}
\hline \multicolumn{2}{c}{ Sacch. cerevisiae and Br. custersianus } & \\
\hline 3-methyl-1-butanol & Linear equation & $\mathrm{r}^{2}$ & $\mathrm{Pr}(>\mathrm{F})$ \\
1vvt/97 & $71.058 \mathrm{t}+20.164$ & 0.9889 & $\mathrm{P}<0.01$ \\
91B/84 & $75.208 \mathrm{t}+108.424$ & 0.9976 & $\mathrm{P}<0.01$ \\
1vvt/97+Brett & $74.629 \mathrm{t}-22.753$ & 0.9968 & $\mathrm{P}<0.01$ \\
91B/84+Brett & $93.698 \mathrm{t}+0.924$ & 0.9812 & $\mathrm{P}<0.01$ \\
2-methyl-1-butanol & Linear equation & $\mathrm{r}^{2}$ & $\mathrm{Pr}(\mathrm{F})$ \\
1vvt/97 & $23.975 \mathrm{t}+5.030$ & 0.9953 & $\mathrm{P}<0.01$ \\
91B/84 & $39.7060 \mathrm{t}+6.2230$ & 0.9998 & $\mathrm{P}<0.01$ \\
1vvt/97+Brett & $26.1540 \mathrm{t}-14.2830$ & 0.9991 & $\mathrm{P}<0.01$ \\
91B/84+Brett & $36.738 \mathrm{t}-27.421$ & 0.9938 & $\mathrm{P}<0.01$ \\
2-methyl-1-propanol & Linear equation & $\mathrm{r}^{2}$ & $\mathrm{Pr}(>\mathrm{F})$ \\
1vvt/97 & $12.227 \mathrm{t}+3.506$ & 0.9846 & $\mathrm{P}<0.01$ \\
91B/84 & $10.3250 \mathrm{t}+20.2000$ & 0.9882 & $\mathrm{P}<0.01$ \\
1vvt/97+Brett & $12.798 \mathrm{t}-2.666$ & 0.9843 & $\mathrm{P}<0.01$ \\
91B/84+Brett & $10.325 \mathrm{t}+10.020$ & 0.9183 & $\mathrm{P}<0.05$ \\
\hline
\end{tabular}

These results showed that the two cultures with the strain 91B/84 did not present significant difference with regard to propanol-1 concentration after six days of tumultuous phase $(\mathrm{P}>0.05)$. In all other cases, the difference was highly significant $(\mathrm{P}<0.01)$. This meant that not only the concentration of this compound but also the interference of $\mathrm{Br}$. custersianus on the production also depended upon the yeast strain. Rankine (1967) showed that the amounts of n-propanol, iso-butanol and iso-amyl plus active amyl alcohol produced during the fermentation of grape juice varied considerably not only according to the yeast but also according to the varieties of grapes (Vitis vinifera) used.

Metabolic response of Sacch. cerevisiae to single and to combined inoculation with $\mathrm{Br}$. custersianus in sterile grape must

The activity of Sacch. cerevisiae was clearly repressed by the presence of Br. custersianus (Fig.
2 and 3). The metabolic inhibition can be better evaluated by the rates of $\mathrm{CO}_{2}$ evolution. An exponential evolution phase was detected between the fourth and the ninth days for both the mixed and pure cultures. The rates of $\mathrm{CO}_{2}$ production are depicted in Table 2. The highest rates of $\mathrm{CO}_{2}$ were obtained with the strain $1 \mathrm{vvt} / 97$ regardless of single, or mixed culture. The inhibition of the metabolism seemed to start five days after inoculation for the strain Sacch. cerevisiae 1vvt/97 (Fig. 2) and after nine days for the strain Sacch. cerevisiae 91B/84 (Fig. 3). Statistical analysis performed after 18 days fermentation showed no significant differences in the $\mathrm{CO}_{2}$ evolution between the two single cultures of Sacch. cerevisiae ( $\mathrm{T} 1$ and $\mathrm{T} 2$ ) and the two mixed cultures (T3 and T4) (P>0.05). Highly significant differences were found in $\mathrm{CO}_{2}$ evolution between the single and mixed cultures ( 11 and $\mathrm{T} 3, \mathrm{~T} 2$ and $\mathrm{T} 3, \mathrm{~T} 1$ and $\mathrm{T} 4, \mathrm{~T} 2$ and $\mathrm{T} 4)(\mathrm{P}<0.01)$. It is well known that Brettanomyces species are ethanol and 
acetic acid producers. As a Custer positive species, $\mathrm{Br}$. bruxellensis is dependent on the level of aeration. Uscanga et al. (2003) showed that the higher the oxygen supply, the greater the acetic acid formation and the lower the ethanol production. Ciani and Ferraro (1996) observed that the absence of an increase in acetic acid in wines did not exclude the presence of Brettanomyces and its high acetic acid production was dependent on the presence of oxygen. The conversion of acetaldehyde to acetic acid is an oxidative process, which results in a drop in $\mathrm{NAD}^{+} / \mathrm{NADH}$ ratio. As a consequence, the glycolytic flux stops at the level of glyceraldehyde-3-phosphate. The acetic acid formation is limited by the availability of $\mathrm{H}$ - acceptors as oxygen, acetoin, acetone and dihydroxyacetone. In anaerobic conditions, the concentration of these organic $\mathrm{H}$-acceptors can be limited; if available, the anaerobic inhibition of fermentation is abolished. These facts explain the weak activity of $\mathrm{Br}$. custersianus when used as pure culture (Fig. 2). Br. custersianus did not affect the Sacch. cerevisiae metabolism only at the beginning of the tumultuous phase of the vinification process, but also during the period when the concentration of sugar was high and ethanol was low. The inhibition process was progressively increased (Fig. 2 and 3) probably due to the ethanol formation with subsequent production of acetic acid.

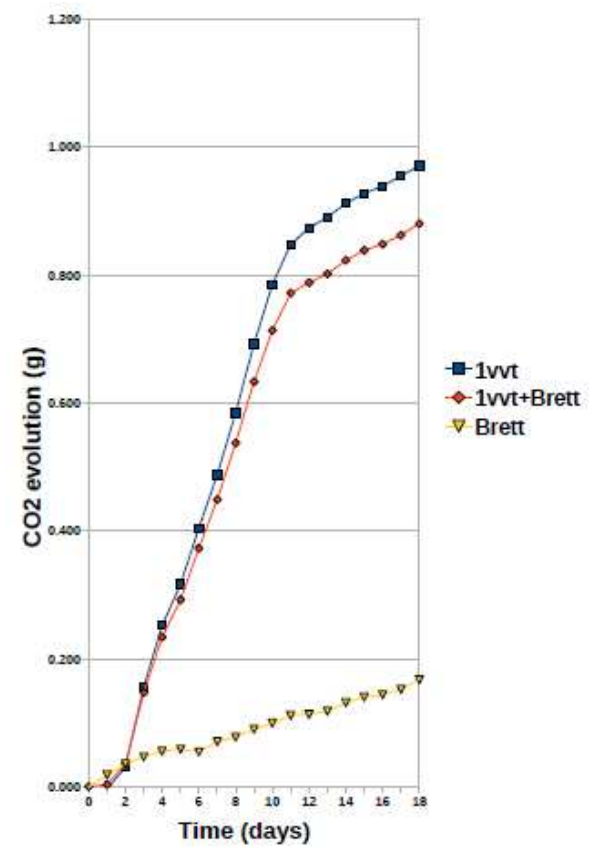

Figure 2 - Yeast activity of Sacch. cerevisiae 1vvt/97 and $\mathrm{Br}$. custersianus measured by $\mathrm{CO}_{2}$ evolution. Single and mixed cultures.

Table 2 - Exponential regressions for $\mathrm{CO}_{2}$ evolution between the fourth and the ninth day of single and mixed fermentations.

\begin{tabular}{llll}
\hline \multicolumn{3}{c}{ Sacch. cerevisiae and Br. custersianus } \\
\hline Strains & Exponential equations & $\mathrm{r}^{2}$ & $\operatorname{Pr}(>\mathrm{F})$ \\
\hline & Single & & \\
$\mathbf{1 v v t / 9 7}$ & $0.1158 \mathrm{e}^{0.202 t}$ & 0.9944 & $\mathrm{P}<0.01$ \\
$\mathbf{9 1 B / 8 4}$ & $0.1219 \mathrm{e}^{0.1778 \mathrm{t}}$ & 0.9961 & $\mathrm{P}<0.01$ \\
& Mixed & & \\
$\mathbf{1 v v t / 9 7 + B r e t t}$ & $0.1082 \mathrm{e}^{0.1998 \mathrm{t}}$ & 0.9944 & $\mathrm{P}<0.01$ \\
$\mathbf{9 1 B / 8 4 + B r e t t}$ & $0.1381 \mathrm{e}^{0.154 \mathrm{t}}$ & 0.996 & $\mathrm{P}<0.01$ \\
\hline
\end{tabular}




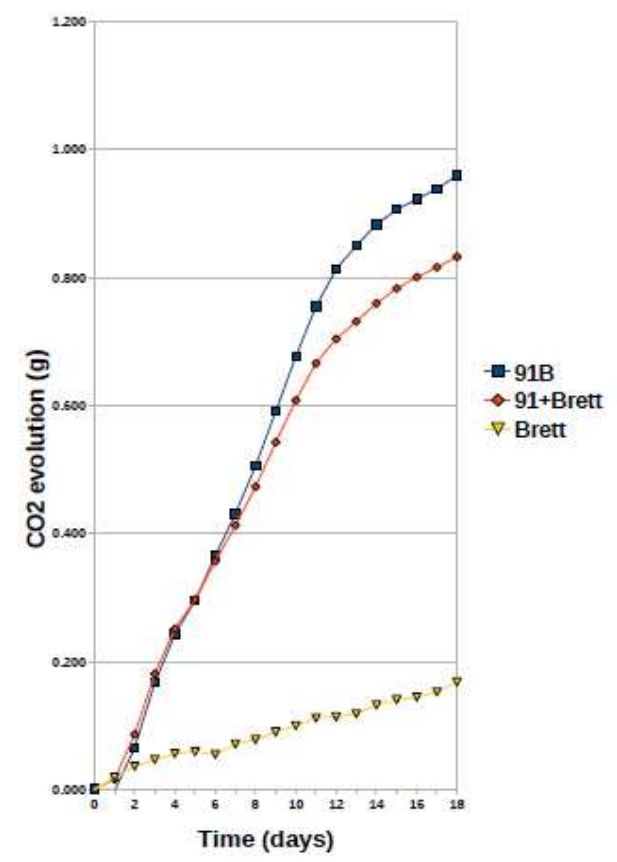

Figure 3 - Yeast activity of Sacch. cerevisiae $91 \mathrm{~B} / 84$ and $\mathrm{Br}$. custersianus measured by $\mathrm{CO}_{2}$ evolution. Single and mixed cultures.

\section{Effect of acetic acid and 4-ethyl-phenol on yeast metabolism}

The addition of acetic acid to the grape must provoked immediate metabolic inhibition of both the Sacch. cerevisiae strains (Fig. 4 and 5). The $1 \mathrm{vvt} / 97$ strain behaved in a fashion similar to $91 \mathrm{~B} / 84$. They showed sensitivity to the lowest concentration of acetic acid used $(2.5 \mu \mathrm{l} / \mathrm{ml})$. As seen from Figures 4 and 5 , at that concentration, the inhibition was acetic acid limited. These results showed that these strains could also be sensitive to other wine-related microorganisms, especially to those acetic acid producers, which were much more commonly found in any phase of the vinification process than were the Brettanomyces species. According to Thomas et al. (2002), it was the total concentration of acetic acid that determined the extent of yeast growth inhibition and not the concentration of undissociated acid alone. Narendranath et al. (2001) showed that the effect of acetic acid depended upon the medium composition and that the length of the lag phase of the growth curve increased exponentially with the increasing concentrations of acetic or lactic acid. It was observed that the length of the lag phase of the $\mathrm{CO}_{2}$ evolution also tended to increase as the concentrations of acetic acid augmented (Fig. 4 and 5). Cássio et al. (2006) demonstrated that acetic acid and some others monocarboxylic acids were competitive inhibitors of DL-lactate transport, suggesting that these acids used similar process of transport of DL-lactate, proton-lactate symport. This transport was only detectable when the medium was depleted in glucose. The uncharged form, however, entered the yeast cell by simple diffusion even in glucose-grown cells. This could lower the intracellular $\mathrm{pH}$, increasing the extrusion of $\mathrm{H}^{+}$(Calahorra et al., 1987) and the ATP expenditure (Eraso and Gancedo, 1987).

These conditions are very stressing to the yeast cells (Dorta et al., 2006). The acetic acid is one of agents that provoke oxidative stress in yeast, inducing apoptosis (Mrozeck and Kufel, 2008). 


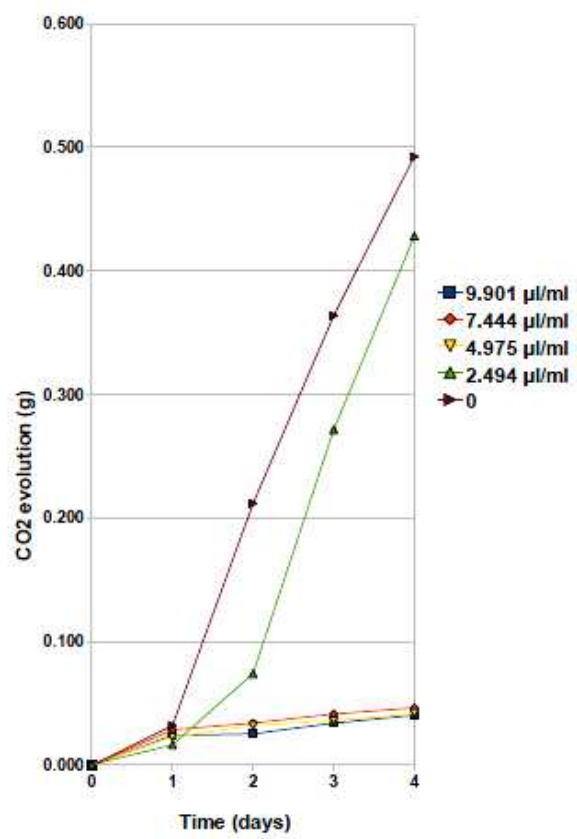

Figure 4 - Inhibition of the Sacch. cerevisiae 1vvt/97 activity provoked by the presence of acetic acid.

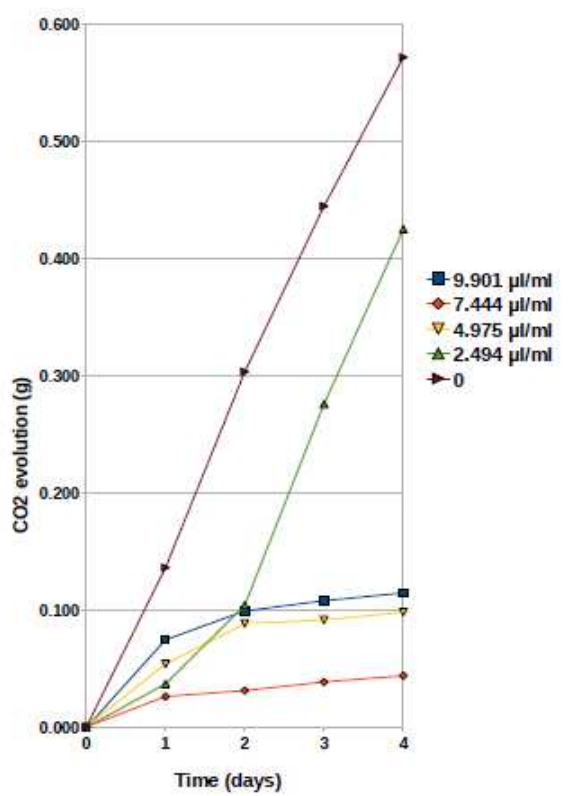

Figure 5 - Inhibition of the Sacch. cerevisiae 91B/84 activity provoked by the presence of acetic acid.

Graves et al. (2006) showed that the inhibition of ethanol production by acetic acid increased as the concentration of solids in the medium increased and the $\mathrm{pH}$ of the medium declined. There are membrane proteins that protect the yeasts against the potential toxicity of weak organic acids secreted by the competitor organisms. One of these proteins was identified as ATP-binding cassette $(\mathrm{ABC})$ transporter Pdr12 (Piper et al., 1998). Piper et al. (2001) suggested that Pdr12 transporter was used to actively transport acid anions or alcohol molecules to the opposite side of the membrane and released them into the aqueous phase of the periplasm. This lowered the 
intracellular level of these compounds. This active transport could explain the lack of total collapse of the two yeast strains activity in must containing $2.5 \mu \mathrm{l} / \mathrm{ml}$ of acetic acid. Maiorella et al. (1983) reported that the acetic acid increased the ATP requirement for maintenance functions, decreased the ratio of specific growth rate/ethanol productivity, disrupted the cell membrane and altered the cell morphology.

The addition of 4-ethyl-phenol to grape must did not affect the metabolism of both the yeasts (Fig. 6 and 7).

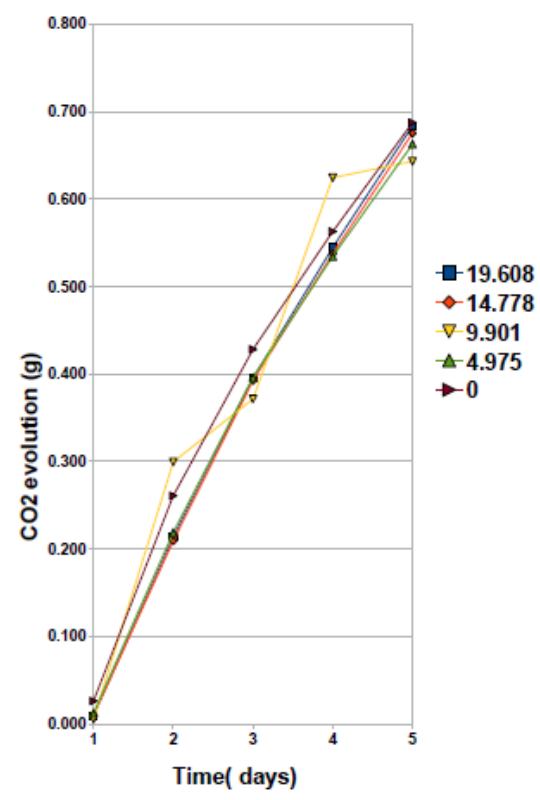

Figure 6 - Activity of Sacch. cerevisiae 1vvt/97 in presence of 4-ethylphenol $(\mu \mathrm{g} / \mathrm{ml})$.

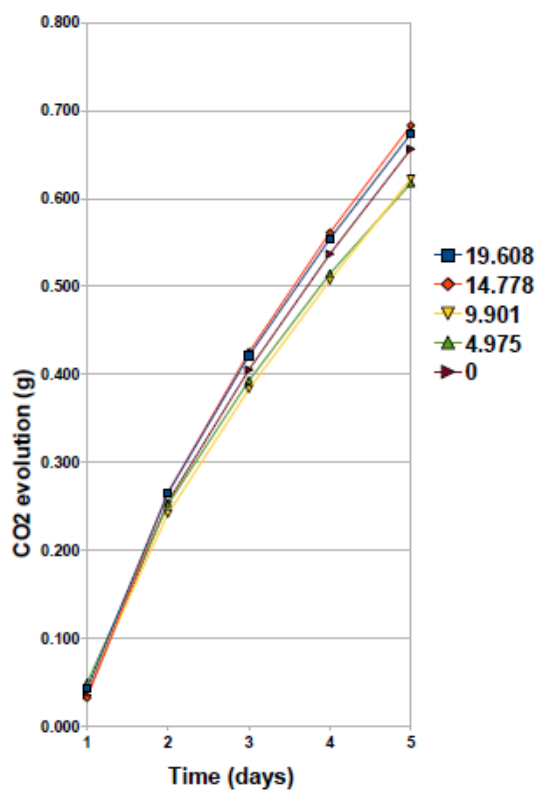

Figure 7 - Activity of Sacch. cerevisiae 91B/84 in presence of 4-ethylphenol $(\mu \mathrm{g} / \mathrm{ml})$.

\section{ACKNOWLEDGEMENT}

The authors thank the $\mathrm{CNPq}$ and Capes for financial support.

\section{REFERENCES}

Bisson, L.F. (1999), Stuck and sluggish fermentations. Am. J. Enol. Vitic., 50, 107-119. 
Calahorra, M., Ramire, J., Clemente, S. M., Peña, A. (1987), Electrochemical potential at ion transport in vesicles of yeast plasma membrane. Biochimica et Biophysica Acta, 899, 229-238.

Cássio, F., Leão, C., van Uden, N. (1987), Transport of lactate and other short-chain monocarboxylates in the yeast Saccharomyces cerevisiae. Appl. Environ. Microbiol., 53, 509-513.

Chatonnet, P., Viala, C., Dubourdieu, D. (1997), Influence of Polyphenolic Components of Red Wines on the Microbial Synthesis of Volatile Phenols. Am. J. Enol. Vitic., 48, 443-448

Ciani, M., Ferraro, L. (1996), Enhanced glycerol content in wines made with immobilized Candida stellata cells. Appl. Environ. Microbiol., 62, 128-132.

Conterno, L., Joseph, C. M. L., Arvik, T. J., Henick-Kling, T., Bisson, L. F. (2006), Genetic and physiological characterization of Brettanomyces bruxellensis strains isolated from wines. Am. J. Enol. Vitic. 57, 139-147.

da Silva, G. A. (1996), The occurrence of killer, sensitive, and neutral yeasts in Brazilian Riesling Italico grape must and the effect of neutral strains on killing behavior. Appl. Microbiol. Biotechnol., 46, 112-121.

da Silva, G. A., de Almeida, E. A. (2006), Production of yellow-green fluorescent pigment by Pseudomonas fluorescens. Braz. Arch. Biol. Technol., 49, 411-419.

Dorta, C., Oliva-Neto, P., de-Abreu-Neto, M. S., NicolauJunior, N., Nagashima, A. I. (2006), Synergism among lactic acid, sulfite, $\mathrm{pH}$ and ethanol in alcoholic fermentation of Saccharomyces cerevisiae (PE-2 and M26). World J. Microbiol. Biotechnol., 22, 177-182

Eglinton, J. M., Henschke, P. A. (1999), Restarting incomplete fermentations: the effect of high concentrations of acetic acid. Australian Journal of Grape and Wine Research, 5, 71-78.

Eglinton, J. M., Henschke, P. A. (1999a) The occurrence of volatile acidity in Australian wines. Australian Grapegrower and Winemaker. 426a, 7-8, 10-12.

Eraso, P., Gancedo, C. (1987), Activation of yeast plasma membrane ATPase by acid $\mathrm{pH}$ during growth. FEBS Letters, 224, 187-192.

Freer, S. N., Dien, B., Matsuda, S. (2003), Production of acetic acid by Dekkera/Brettanomyces yeasts under conditions of constant pH. World J. Microbiol. Biotechnol., 19, 101-105.

Giudici, P., Zambonelli, C. (1992), Biometric and Genetic Study on Acetic Acid Production for Breeding of Wine Yeast. Am. J. Enol. Vitic., 43, 370-374.

Graves, T., Narendranath, N. V., Dawson, K., Power, R. (2006), Effect of $\mathrm{pH}$ and lactic or acetic acid on ethanol productivity by Saccharomyces cerevisiae in corn mash. J. Ind. Microbiol. Biotechnol., 33, 469-474.

Heresztyn, T. (1986), Formation of substituted tetrahydropyridines by species of Brettanomyces and Lactobacillus isolated from mousy wines. Am. J. Enol. Vitic., 37, 127-132.
Logan, M. (2005), R and S-PLUS: Basic instructions Monash University. School of Biological Sciences

Maiorella, B., Blanch, H. W., Wilke, C. R. (1983), Byproduct inhibition effects on ethanolic fermentation by Saccharomyces cerevisiae. Biotechnol. Bioeng. 25, 103121.

Mroczek, S., Kufel, J. (2008), Apoptotic signals induce specific degradation of ribosomal RNA in yeast. Nucleic Acids Res., 36, 2874--2888

Narendranath, N. V., Thomas, K. C., Ingledew, W. M. (2001), Effects of acetic acid and lactic acid on the growth of Saccharomyces cerevisiae in a minimal medium. J. Ind. Microbiol. Biotechnol., 26, 171-177.

Piper, P., Mah, Y., Thompson, S., Pandjaitan, R., Holyoak, C., Egner, R., Mhlbauer, M., Coote, P., Kuchler, K. (1998), The Pdr12 ABC transporter is required for the development of weak organic acid resistance in yeast. EMBO J., 17, 4257-4265.

Piper, P., Calderon, C. O., Hatzixanthis, K., Mollapour, M. (2001), Weak acid adaptation: the stress response that confers yeasts with resistance to organic acid food preservatives. Microbiology, 147, 2635-2642.

Querol, A., Jimnez, M., Huerta, T. (1990), Microbial and enological parameters during fermentation of must from poor and normal grape-harvest in the region of Alicante. J. Food Sci., 55, 1603-1606.

Rankine, B. C. (1967), Formation of higher alcohols by wine yeasts. J. Sci. Fd Agric., 18, 583-58.

Renouf, V., Lonvaud-Funel, A. (2007), Development of an enrichment medium to detect Dekkera/Brettanomyces bruxellensis, a spoilage wine yeast, on the surface of grape berries. Microbiol. Res., 162, 154-167.

Ribereau-Gayon, J., Peynaud, E., Sudraud, P., RibereauGayon, P. (1982), Traité d'Enologie. Siences et techniques du vin. Analyse et contrôle des vins, Dunod, Paris.

Scheffers, W. A., Wiken, T. O. (1969), The Custers effect (negative Pasteur effect) as a diagnostic criterion for the genus Brettanomyces. Antonie Van Leeuwenhoek, 35, A31-A32.

Silva, P., Cardoso, H., Géros, H. (2004), Studies on the Wine Spoilage Capacity of Brettanomyces/Dekkera spp. Am. J. Enol. Vitic., 55, 65-72.

Thomas, K. C., Hynes, S. H., Ingledew, W. M. (2002), Influence of medium buffering capacity on inhibition of Saccharomyces cerevisiae growth by acetic and lactic acids. Appl. Environ. Microbiol., 68, 1616-1623

Uscanga, M. G. A., Dlia, M-L., Stehaiano, P. (2003), Brettanomyces bruxellensis: effect of oxygen on growth and acetic acid production. Appl. Microbiol. Biotechnol., 61, 157-162.

Venables, W. N., Smith, D. M., the R Development Core Team (2004), An introduction to R. The R Development Core Team, edition 2.0.1.

Received: July 27, 2009; Revised: October 22, 2010; Accepted: February 02, 2011. 